UWS Academic Portal

\title{
A comparative biomechanical analysis of the performance level on chasse step in table
} tennis

Yu, Changxiao; Shao, Shirui; Baker, Julien S.; Awrejcewicz, Jan; Gu, Yaodong

Published in:

International Journal of Sports Science and Coaching

DOI:

$10.1177 / 1747954119843651$

Published: 01/06/2019

Document Version

Peer reviewed version

Link to publication on the UWS Academic Portal

Citation for published version (APA):

Yu, C., Shao, S., Baker, J. S., Awrejcewicz, J., \& Gu, Y. (2019). A comparative biomechanical analysis of the performance level on chasse step in table tennis. International Journal of Sports Science and Coaching, 14(3), 372-382. https://doi.org/10.1177/1747954119843651

\section{General rights}

Copyright and moral rights for the publications made accessible in the UWS Academic Portal are retained by the authors and/or other copyright owners and it is a condition of accessing publications that users recognise and abide by the legal requirements associated with these rights. 
Reprinted by permission of SAGE Publications. 

8 9 0

\section{A comparative biomechanical analysis of the performance level on chasse step in table tennis}

\section{Changxiao Yua, Shirui Shao ${ }^{a}$, Julien S Baker ${ }^{b}$, Jan Awrejcewicz ${ }^{c}$, Yaodong $\mathbf{G u}^{\mathrm{a} *}$}

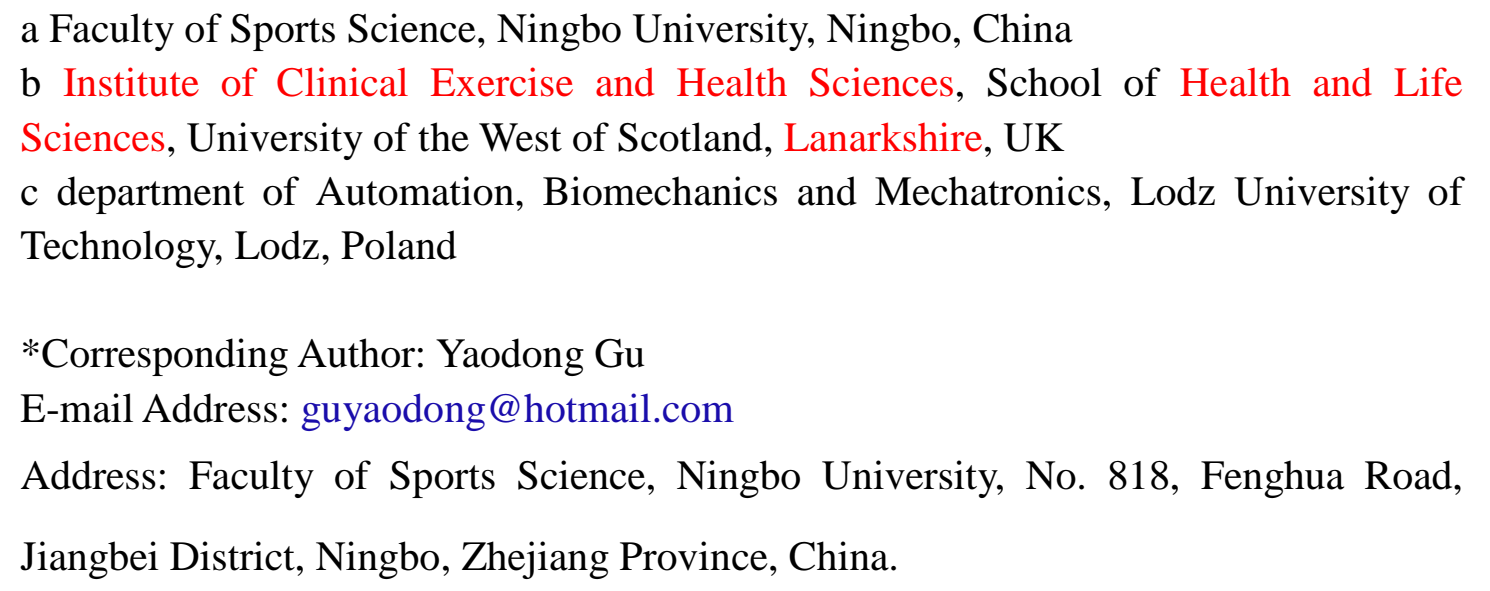




\section{A comparative biomechanical analysis of the performance level on chasse step in table tennis}

\section{Abstract}

Initial observations have indicated that each movement pattern or skill set has a fundamental mechanical structure. The purpose of this study was to examine biomechanical characteristics in chasse step movement patterns between professional athletes (PA) and beginner players (BP). Large amounts of data were obtained for comparison by capturing kinematic and kinetic information of the dominant foot using the Oxford Foot Model (OFM) during table tennis strokes. Nine male PA and nine BP (all with dominant right feet) participated in a table tennis footwork test. A Vicon motion analysis system and a Novel Pedar insole plantar pressure measurement system were used to record kinematic and kinetic data, respectively. Findings from the study indicated that PA not only showed significantly larger forefoot and rear-foot dorsiflexion, but also demonstrated larger hallux plantarflexion. In addition, they also showed significantly larger forefoot abduction and rear-foot internal rotation than BP at the chasse step end. Also, PA showed significantly larger forefoot inversion and abduction at the forward-end of the step. Peak pressure values were higher under the lateral forefoot, and the medial and lateral rear-foot with faster changes in angular velocity recorded for PA during the chasse step phase. Greater peak pressures were also recorded under the other toes, and in the central and lateral forefoot during the forward swing phase when compared to BP. In addition, PA showed significantly greater relative load on the other toes and on the lateral forefoot during the entire step motion. The results of the present study demonstrated that PA possessed greater foot drive technique. These findings might help coaches and beginners to comprehend the internal mechanisms of the chasse step technique and help influence beginner players to improve the mechanical efficiency of performance.

Keywords: Oxford Foot Model, footwork, internal mechanisms, lower limb drive 


\section{Introduction}

Table tennis is not only a complex and asymmetric sport, but is also one of the most popular sporting activities worldwide. According to a recent report by the International Sports Federation (ISF), the population of table tennis participants has reached over 300 million in the world [34]. Playing table tennis is regarded as a pro-health sporting pastime, which is generally accepted by more and more people who engage in physical activity [3]. As a rational movement, agile footwork enables a player to rapidly change his/her position/direction and regulate his/her body for a particular stroke with maximal power and effect [22]. In addition, footwork is the fundamental closed skill that requires active movement and accurate control. Therefore, table tennis footwork could provide an appropriate link for maximizing stroke performance. There are five types of table tennis footwork that have been identified: one step, chasse step, slide step, cross step and turn step. The chasse step - if the ball is presented at the right side, the right foot is moved to the right in the first instance, followed by the left foot in a sliding action in the same direction - and is considered as one of the most frequently used attacking footwork movement patterns with a frequency of $15.2 \%$ in competitions [20]. As a complex task, table tennis requires a command of the necessary motor skills for the beginner to become proficient and progress through the developmental period [16]. The proper footwork is regarded as one part of the technical skills that can reflect the players' level [2]. The comparison of basic motion patterns between the professional athletes (PA) and beginner players (BP) using a specific foot model can be a valuable tool in understanding the internal mechanisms involved in leaning the chasse step and identifying the key factors underlying effective performance. Despite obvious differences that exist between PA and BP related to the lower limb contribution during the chasse step, coordinated motion patterns that produce better accuracy and faster connections with the next stage of performance, are of great importance for both the coaches and athletes.

The control of footwork patterns during table tennis performance requires a coordinated sequence of body segment interactions, and the optimum activation of all 
the links has been defined as the "kinetic chain” [14], [17]. A forceful lower limb drive is considered as the "starting point" of the kinetic chain in sports [10]. The starting point is influential in the quality of stroke produced and is very important in the development of skills that influence match play (racquet and ball speed and a high degree of accuracy, etc.) [13], [14]. The lower limb is the source of energy that transfers optimum activation from the lower body segments to the upper limb through sequential movements of the kinetic chain [10], [24]. Therefore, high quality table tennis footwork not only requires greater upper limb co-ordination, but also needs support from the lower limbs' to provide accuracy and stability during competition and training. The powerful lower limb drive could contribute to increasing the translational and spinning velocity of the ball. Research by Qian et al. [24] concluded that the differences in motion of the intrinsic joints of the lower limb could influence the velocity of the table tennis bat during the table tennis forehand loop between the superior players and intermediate players.

A number of generic musculoskeletal models of the lower limb have been proposed to analyze the large joints such as the hip, knee and ankle. However, small joint musculoskeletal models of the foot were almost unobtainable until the development of the Oxford Foot Model (OFM). The lower-limb model using the OFM provides an increased level of detail in the foot, and it is a reliable multi-segment investigative foot analysis tool. Previous research has tended to focus on the analysis of the forefoot, hind foot and hallux motions [5], [19]. During a step forward task analysis, and other movement patterns, the model can be widely used in the motion analysis of performers in both sport and clinical environments. For initial analysis of foot models, marker placement error is a fundamental challenge due to the lack of rigorous anatomical coordinate definition. This could cause large errors in angular calculations even though the errors in marker locations are minimal [8], [21], [26]. Considering the characteristics of table tennis, players have to complete a series of complex spatial movements that include, acceleration, deceleration, direction change, moving quickly and balance control. All of these varied and different movement patterns help generate optimum stroke production [12]. Therefore, in order to study table tennis footwork, rigorous 
research should be conducted using a reliable and objective foot model. As a multi-segment kinematic model, the OFM has recorded higher accuracy compared to other methodologies and has been used to measure inter-segmental angles of the foot [7]. The methodologies used have been applied to a wide range of populations with different abilities [19], [31]. The OFM, however, has not been used to evaluate the footwork patterns of table tennis beginners. Therefore, the aim of this study was to compare the differences in forefoot, hind foot and hallux motions between PA and BP using the OFM during the chasse step movement patterns. Additionally, the use of the OFM will be used in conjunction with data obtained from a Visual three-dimensional (V3D). This will provide additional information to compare and contrast the chasse step movement patterns. This will be essential for detecting the internal mechanisms of lower limb movements between the two different levels of athletes participating in table tennis.

Further to this, table tennis is a classic sport that typically needs upper limb, lower limb and abdominal simultaneous contractions to complete stroke performance instantaneously. The powerful forward swing of the upper limb demands the support from a forceful lower limb, and as the origin of the energy force, the foot drive impacts the accuracy and quality of strokes. Therefore, the "Foot (shoe)-ground" biomechanical characteristics should be taken into account. In order to investigate the mechanical basis of table tennis footwork patterns, both for better understanding of the mechanisms and performance improvement, the ability to use a Novel Pedar insole plantar pressure measurement system is essential. This system can provide accurate information about plantar pressure distribution patterns, compared to a force plate [13]. In addition, as a kinetic measuring method, the system has been applied successfully to study the characteristics of plantar kinetics in different sports. These include different soccer-specific movements of soccer players [9, 25, 28, 30], two types of tennis serve [13] and a comparison of the center of pressure (COP) trajectory in two different levels of table tennis players during topspin forehand loop [11] as well as the dynamic balance in table tennis [15]. 
Our primary aim was to compare the biomechanical characteristics of the chasse step between PA and BP, the findings would provide useful suggestions for coaches and athletes and contribute to an in-depth understanding of the performance characteristics. It was hypothesized that PA would show different plantar foot joint angles from BP at the two key technical areas, respectively. PA would also show larger joint angular changing rate (ACR) with a smaller joint range of motion (ROM) during chasse step. We also hypothesized that there would be significant differences for the two groups at the forward and swing phases during table tennis performance. In addition, we also wanted to examine if any differences in force distribution of in-shoe loading occurred between PA and BP during the entire motion phase.

\section{Material and Methods}

\section{Participants}

Eighteen male right-handed table tennis players from Ningbo University table tennis team (9 professional athletes age $23.5 \pm 1.24$ years, weight $74.68 \pm 2.54 \mathrm{~kg}$, height 1.75 $\pm 0.05 \mathrm{~m}$, training experience $14.8 \pm 1.57$ years; 9 beginners, age $22.7 \pm 1.62$ years, weight $73.75 \pm 3.1 \mathrm{~kg}$, height $1.75 \pm 0.04 \mathrm{~m}$, training experience $0.45 \pm 0.42$ years) were recruited into the study. Ethical approval for the study was obtained from the Ethics Committee of Ningbo University. All participants were informed of experimental procedures and requirements. All were free from any previous lower limb injuries, surgeries or foot diseases six months prior to experimental data collection.

In order to determine the dominant lower limb, the ball-kick test was adopted in this study [33]. In this test, each participant was asked to kick a football with arbitrary power and maximal accuracy through a set of obstacles placed $1 \mathrm{~m}$ apart and $10 \mathrm{~m}$ from the participants, the supporting leg was regarded as the non-dominant limb and the other side was the dominant limb when kicking the football. In addition, this study followed 
the suggestions of Peters and Murphy [23] to determine the dominant hand, and selected right-handed athletes as the participants.

\section{Instrumentation}

Foot kinematics were captured using an eight-camera Vicon motion analysis system (Oxford Metrics Ltd., Oxford, UK) with a frequency of $200 \mathrm{~Hz}$. In order to assess the three-dimensional motion of the lower limb, reflective markers (diameter: $14 \mathrm{~mm}$ ) were used to define joint centre and motion axes in accordance with the OFM marker set. The marker locations included: for the forefoot segment, markers were placed on the head and base of the $1^{\text {st }}$ metatarsal and the $5^{\text {th }}$ metatarsal respectively, the base of the hallux, and the proximal end of the distal phalanx of the $1^{\text {st }}$ metatarsal. The rear-foot segment was defined by placing markers on the medial malleolus, superior heel, posterior calcaneus wand marker, sustentaculum tali, inferior heel and lateral calcaneus. To define the tibial segment, markers were placed on the head of the fibula, tibial tuberosity, lateral shank, anterior aspect of the shin and lateral malleolus (Fig. 1). Kinetic data was recorded by the in-shoe plantar pressure measurement system (Novel GmbH, Munich, Germany) and was recorded at $50 \mathrm{~Hz}$. As outlined in Fig. 6, the plantar was divided into nine anatomical areas including, medial rear-foot (MR), lateral rear-foot (LR), medial mid-foot (MM), lateral mid-foot (LM), medial forefoot (MF), central forefoot (CF), lateral forefoot (LF), hallux (H), and other toes (OT). Prior to testing, the pressure insoles had been regulated with a pressure pump and data recording was sampled through Bluetooth technical equipment. Kinematic and kinetic tests were conducted synchronously. In addition, a high-speed camera (Fastcam SA 3, Photron, Japan) was employed to record the entire motion with a frame rate of $1000 \mathrm{~Hz}$.

\section{Insert Fig. 1. Oxford Foot Model marker placement}

\section{Experimental setup}

All tests and experiments evaluating table tennis footwork were conducted at Ningbo University table tennis training gymnasium. Prior to data collection, all participants were given a standardized warm-up of $20 \mathrm{~min}$ in the experimental environment. All 
participants were required to wear unified training footwear that contained Pedar insoles. Subjects were fully familiarized with the insoles in preparation for data collection. Participants practiced multi-ball training for 10 min using the chasse step. During the test, each participant was first required to return the ball back at the neutral position (Fig. 2, a-c). Using the chasse step movement, then they were asked to perform a single forehand loop, accurately at a $0.15 \times 0.15 \mathrm{~m}$ target area bordering the net of the right serve box at match pace, with maximal power against a topspin ball that was projected by a table tennis ball machine placed $1.3 \mathrm{~m}$ away from the player' court (Fig. 2, d-f). The devices used did not influence or interfere with any of the recorded motions.

\section{Insert Fig. 2. The technique performance of the participant during the test}

\section{Data processing}

A complete motion of the chasse step was recorded from the former stroke end to the backswing end, during the backswing phase. In order to study the motion in detail, two distinct phases of the table tennis performance were observed backswing phase (I) and forward swing phase (II). Moreover, in this study we selected two key events (backward-end, BE; forward-end, FE) from the entire motion. All joint angles in three planes were time-normalized to 100 data points. Kinematic analyses of the two stages were conducted on the following dependent variables: ROM and ACR. As kinetics parameter, peak pressures were determined for the nine selected areas. In addition, the force-time in each foot region was calculated and defined as the relative load for the individual region [9], [13].

\section{Statistical analysis}

SPSS 19.0 (SPSS Inc., Chicago, IL, USA) was utilized for statistical analysis. Prior to statistical analysis, Shapiro-Wilks normality test was conducted to ensure that all data sets were normally distributed. Between group differences in kinematics, kinetics and time variables were evaluated using independent $t$-tests. The significance level for all tests was set at $p<0.05$. Cohen's $d$ was used to compare the differences in the average of the two groups, and it is often represented by the effect size [6]. Effect size (ES) is 
evaluated as trivial $(\geq 0.19)$, small $(\geq 0.2$ and $\leq 0.49)$, medium $(\geq 0.50$ and $\leq 0.79)$ and large $(\geq 0.80)$, respectively [6].

\section{Results}

\section{Time}

The time to perform each phase for PA and BP is shown in Table 1 with significance levels, respectively. Compared with BP, PA showed significantly shorter time to compete the phase I and demonstrated longer time to complete phase II.

Insert Table 1. Mean \pm standard deviations (Mean \pm SD), standard error of measurement (SEM), 95\% confidence intervals (CI), effect sizes (ES) for the time at phase I, phase II

\section{Insert Fig. 3. Changes of plantar foot joints during one motion cycle in three planes}

\section{Kinematics}

The changes of plantar foot joints are shown as Fig. 3, which were generally comparable for both PA and BP in the three planes during one entire motion cycle. As in Table 2 and 3, significant kinematic differences in all three planes of RHFTBA (right hindfoot with respect to tibia angles), RFFHFA (right forefoot with respect to hindfoot angles) and RHXFFA (right hallux with respect to forefoot) have been detected between phase I and II in the two groups. The details as listed: (1) compared with BP, PA demonstrated significantly greater rear-foot dorsiflexion, smaller rear-foot inversion and greater rear-foot internal rotation at BE. (2) PA demonstrated significantly greater forefoot dorsiflexion, smaller forefoot eversion and greater forefoot abduction with greater hallux plantarflexion compared to BP at BE. (3) At FE, PA showed increased rear-foot dorsiflexion and internal rotation with greater forefoot inversion and abduction than BP. For the BP, ROM of the plantar foot joints in the three planes showed to be significantly larger at phase I (Table 4), while it was significantly smaller than the PA at phase II (Table 5). Concerning ACR of plantar foot joints, ACR at the RHFTBA for PA 
showed significantly larger in the sagittal and frontal planes, while ACR at RHXFFA was significantly smaller than BP during phase I (Fig. 4). Compared with BP, ACR at the RFFHFA for PA represented significantly larger in the frontal plane during the entire motion (Fig. 4).

Insert Table 2. Mean \pm standard deviations (Mean \pm SD), standard error of measurement (SEM), 95\% confidence intervals (CI), effect sizes (ES) for the comparison of plantar foot joint angles at key events in three planes between the PA and BP at the phase I

Insert Table 3. Mean \pm standard deviations (Mean \pm SD), standard error of measurement (SEM), 95\% confidence intervals (CI), effect sizes (ES) for the comparison of plantar foot joint angles at key events in three planes between the PA and BP at the phase II

Insert Table 4. Mean \pm standard deviations (Mean \pm SD), standard error of measurement (SEM), 95\% confidence intervals (CI), effect sizes (ES) for the plantar foot joints ROM of the motion of PA and BP at the phase I

Insert Table 5. Mean \pm standard deviations (Mean \pm SD), standard error of measurement (SEM), 95\% confidence intervals (CI), effect sizes (ES) for the plantar foot joints ROM of the motion of PA and BP at the phase II

Insert Fig. 4. Angular changing rate of plantar foot joints (upper: PA; lower: BP) during one entire motion in three planes

\section{Kinetics}

Peak pressures were higher under the LF, MR and LR areas in PA than BP, while the regions of the $\mathrm{H}, \mathrm{MF}$ and $\mathrm{CF}$ were shown to be significantly smaller for PA at phase I (Fig. 5). Higher peak pressure was observed on the OT, LF and CF areas for PA, but the $\mathrm{H}$ area was smaller than BP during phase II (Fig. 5). In general, relative loads were higher on the OT and LF areas but lower on the MF and MR areas for PA compared with BP (Fig. 6). No main effect was observed for peak pressure and relative load between the PA and BP at MM and LM areas.

Insert Fig. 5. Peak pressure for phase I and II between the PA and BP in each of the nine 
areas of interest

Insert Fig. 6. Mean and standard deviation relation load (\%) for dominant foot between the $P A$ and $B P$ in each of the nine areas of interest during one entire motion

\section{Discussion}

The main aim of this experiment was to study the differences of foot biomechanics between PA and BP during the chasse step. The analysis of foot mechanics in some sports has been a major challenge for many years. Based on the moderate scientific evidence, qualitative interpretations are most often used for current paradigms and concepts of foot functions in general. Combining with OFM, the V3D technology can measure in vivo foot kinematics to solve many practical questions. Two key phases (chasse step and forward swing) were identified for in-depth analysis. A thorough understanding of lower-limb movement patterns of different level players has important implications on the enhancement of technical performance and the prevention of potential sport injuries. The findings of this study indicate that there were significant differences in the three-plane movement of the dominant foot between PA and BP. For BP, greater ROM were found in phase I, while it was smaller in phase II compared with PA. In addition, for PA, the joints ACR of RHFTBA during phase I increased significantly in the sagittal and frontal planes than BP, and ACR at RFFHFA showed to be larger during the entire motion. Significant differences in kinetics were found in LF, MF and LR areas in peak pressure between PA and BP during phase I. With respect to plantar relation load of the entire motion, significant differences were observed in OT, ME, LF and MR areas between PA and BP. When performing the chasse step, PA could take less time than BP. However, forward swing was executed in more time, which suggests that PA had enough time to accelerate the racket rapidly and regulate the body balance. 
Based on the observation of significantly smaller ROM for PA at the completion of chasse step compared with BP, it can be inferred that PA possessed a stronger ability of using foot control and better technological stabilization. Rear-foot movement of PA progressed to obvious internal rotation at BE, it could help players to better finish the chasse step motion. At the same time, the greater forefoot abduction would be of great benefit to maintain body balance. In addition, using high speed camera and V3D, we found that PA were accustomed to landing on the rear-foot area while BP were at forefoot area during phase I. This was in line with the observation that the peak pressure of PA was higher under MR, LR and LF areas, in contrast, that of BP was higher on $\mathrm{H}$, MF and CF areas during this period. This suggests that PA were able to distribute body weight more evenly on the full plantar to provide a more stable base for the next phase. At the event of BE, PA showed significantly smaller rear-foot inversion and forefoot eversion as well as larger hallux plantarflexion than BP, it also contributed to greater balance and was a potential factor to rapidly link the next stage of the kinetic chain. All of the results of this study indicate that PA possessed a more stable center of mass shift for the chasse step. As expected, significant differences were observed between PA and $\mathrm{BP}$ in rear-foot and forefoot dorsiflexion. Based on the theory of the stretch-shortening cycle, that states prior stored elastic energy in a muscle-tendon stretching phase could increase concentric movement [10], [29], it can be suggested that PA presented a fuller-backswing for the increased dorsiflexion that may enhance muscle output for performing the following motion. The same standpoint was confirmed by Komi et al. [18], they found that there was significant elastic energy storage for a squat jump compared with drop jump and counter-movement jump at the starting position. Interestingly, in order to assess the ability of an efficient energy transfer in the kinetic chain, the faster joint angular velocity is considered as standard and can reflect the level of technical movements [24], [27]. According to the comparison in ACR of plantar foot joints during phase I between PA and BP, PA showed was faster for RHFTBA in the sagittal and frontal planes. Meanwhile, the ACR at RFFHFA for PA was remarkably larger in the frontal plane than that of BP at the same moment. The increased ACR of 
RHFTBA and RFFHFA for PA in this study may be related to more skilled technique and more effective plantar foot drive during chasse step.

With longer time in phase II, PA showed greater ROM of plantar foot joints. Previous studies have corroborated that experienced racquet-sport players have a better anticipation ability that can predict the outcomes of the movements of their opponents and even the ball trajectory [1], [4], [32], [35]. Based on this finding, it can be speculated that PA possessed a better ability of flexible motions than BP which would be beneficial for themselves to improve the quality of stroke. Additionally, compared with BP at the event of FE, PA showed larger rear-foot internal rotation and forefoot abduction. This may suggest a more fully forward swing to enhance the racquet speed for PA when synthesizing the observations of greater peak pressure under OT, LF and CF at the period. The increased rear-foot dorsiflexion with greater forefoot inversion of PA at FE moment may lead to quick return back to the starting position. The results of the present study indicated that both the lateral and the medial area of the forefoot were loaded differently, due to the different level of performance. For PA, higher relative load was recorded on the OT and LF areas compared with BP - MF and MR areas showed higher relative load for BP - during one entire motion cycle.

There are some limitations that should be noted in this study. Firstly, due to restrictions in enrollment, this study lacked of the comparison of the relationship between skill level and gender in chasse step. Another potential limitation is that there were substantial differences in training years, although all PA had same skill level and they were from same university table tennis team, this may limit the external validity at some degree. Further, BP possibly had different levels of cognitive ability in nature, which could influence the degree of learning. Finally, the generalization and application of these findings to players from other countries may be treated cautiously, due to the fact that the participants in this study were from China. 
This study aimed to investigate the differences in the biomechanical characteristics during the chasse step between professional athletes and beginner players. As the results of this study indicated, professional athletes performed the chasse step within less time, and were coupled with significantly smaller ROM of plantar foot joint as well as significantly higher angle changing rate at forefoot and rear-foot compared with beginner players. It confirmed that professional athletes possessed better ability of using foot drive in this technique. In addition, the significantly smaller forefoot eversion and rear-foot inversion as well as greater hallux plantarflexion were recorded for professional athletes, which is a possible strategy to maintain body stability at the backswing end. For professional athletes at the forward swing phase, higher peak pressure under OT, LF and CF areas were recorded. Additionally, relative load was higher on the OT and LF areas for professional athletes compared with beginner players. These results could provide quantitative evidence for coaches and beginner players of how to better manipulate foot motions for improving the quality of the chasse step and to increase the control of body stability during the entire motion period. 


\section{References}

404

405

406

407

408

409

410

411

412

413

414

415

416

417

418

419

420

421

422

423

424

425

426

427

428

429

430

431

432

433

434

435

436

[1] Aglioti SM, Cesari P, Romani M, et al. Action anticipation and motor resonance in elite basketball players. Nat Neurosci 2008; 11(9): 1109.

[2] Bańkosz Z and Winiarski S. Correlations between angular velocities in selected joints and velocity of table tennis racket during topspin forehand and backhand. $J$ Sports Sci Med 2018; 17(2): 330.

[3] Biernat E, Buchholtz S and Krzepota J. Eye on the ball: table tennis as a pro-health form of leisure-time physical activity. Int J Environ Res 2018; 15(4): 738.

[4] Brown EC and Brüne M. The role of prediction in social neuroscience. Front Hum Neurosci 2012; 6: 147.

[5] Carson MC, Harrington ME, Thompson N, et al. Kinematic analysis of a multi-segment foot model for research and clinical applications: a repeatability analysis. J Biomech 2001; 34(10): 1299-1307.

[6] Cohen J. Statistical power analysis for the behavioral sciences (Rev. ed). New York: Academic Press, 1988.

[7] Curtis DJ, Bencke J, Stebbins JA, et al. Intra-rater repeatability of the Oxford foot model in healthy children in different stages of the foot roll over process during gait. Gait Posture 2009; 30(1): 118-121.

[8] Dul J and Johnson GE. A kinematic model of the human ankle. J Biomed Eng 1985; 7(2): 137-143.

[9] Eils E, Streyl M, Linnenbecker S, et al. Characteristic plantar pressure distribution patterns during soccer-specific movements. Am J Sports Med 2004; 32(1): 140-145.

[10] Elliott B. Biomechanics and tennis. Br J Sports Med 2006; 40(5): 392-396.

[11] Fu F, Zhang Y, Shao S, et al. Comparison of center of pressure trajectory characteristics in table tennis during topspin forehand loop between superior and intermediate players. Int J Sports Sci Coach 2016; 11(4): 559-565.

[12] Girard O and Millet GP. Neuromuscular fatigue in racquet sports. Phys Med Rehabil Clin N Am 2009; 20(1): 161-173.

[13] Girard O, Eicher F, Micallef JP, et al. Plantar pressures in the tennis serve. $J$ Sport Sci 2010; 28(8): 873-880.

[14] Girard O, Micallef JP and Millet GP. Lower-limb activity during the power serve in tennis: effects of performance level. Med Sci Sports Exerc 2005; 37(6): 1021-1029. [15] Gu Y, Yu C, Shao S, et al. (2019). Effects of table tennis multi-ball training on dynamic posture control. PeerJ 2019; 6: e6262. 
[16] Muelling K, Boularias A, Mohler B, et al. Learning strategies in table tennis using inverse reinforcement learning. Biol Cybern 2014; 108(5): 603-619.

[17] Kibler WB and Van Der Meer D. Mastering the kinetic chain. In: Roetert P and Groppel J, eds. World-Class Tennis Technique. Champaign: Human Kinetics, 2001, pp. 99-113.

[18] Komi PV and Bosco C. Utilization of stored elastic energy in leg extensor muscles by men and women. Med Sci Sports Exerc1978; 10(4): 261-265.

[19] Levinger P, Murley GS, Barton CJ, et al. A comparison of foot kinematics in people with normal-and flat-arched feet using the Oxford Foot Model. Gait Posture 2010; 32(4): 519-523.

[20] Malagoli Lanzoni I, Lobietti R and Merni F. Footwork techniques used in table tennis: a qualitative analysis. In: Proceedings Book of the 10th ITTF Sports Science Congress (Eds.), 2007, pp. 401-408. Zagreb: University of Zagreb.

[21] Moseley L, Smith R, Hunt A, et al. Three-dimensional kinematics of the rearfoot during the stance phase of walking in normal young adult males. Clin Biomech 1996; 11(1): 39-45.

[22] Nikolić I, Furjan-Mandić G and Kondrič M. The relationship of morphology and motor abilities to specific table tennis tasks in youngsters. Coll Antropol 2014; 38(1): 241-245.

[23] Peters M and Murphy K. Cluster analysis reveals at least three, and possibly five distinct handedness groups. Neuropsychologia 1992; 30(4): 373.

[24] Qian J, Zhang Y, Baker JS, et al. Effects of performance level on lower limb kinematics during table tennis forehand loop. Acta Bioeng Biomech 2016; 18(3): 149. [25] Queen RM, Haynes BB, Hardaker WM, et al. Forefoot loading during 3 athletic tasks. Am J Sports Med 2007; 35(4): 630-636.

[26] Saraswat P, Macwilliams BA and Davis RB. A multi-segment foot model based on anatomically registered technical coordinate systems: method repeatability in pediatric feet. Gait Posture 2012; 35(4): 547-555.

[27] Seeley MK, Funk MD, Denning WM, et al. Tennis forehand kinematics change as post-impact ball speed is altered. Sport Biomech 2011; 10(4): 415-426.

[28] Sims EL, Hardaker WM and Queen RM, Gender differences in plantar loading during three soccer-specific tasks. Br J Sports Med 2008; 42(4): 272-277.

[29] Walshe AD, Wilson GJ and Ettema GJ. Stretch-shorten cycle compared with isometric preload: contributions to enhanced muscular performance. J Appl Physiol 1998; 84(1): 97-106.

[30] Wong PL, Chamari K, Chaouachi A, et al. Difference in plantar pressure between the preferred and non-preferred feet in four soccer-related movements. Br J Sports Med 2007; 41(2): 84-92.

[31] Wright CJ, Arnold BL, Coffey TG, et al. Repeatability of the modified Oxford foot model during gait in healthy adults. Gait Posture 2011; 33(1): 108-112.

[32] $\mathrm{Xu} \mathrm{H}$, Wang $\mathrm{P}$, Ye $\mathrm{ZE}$, et al. The role of medial frontal cortex in action anticipation in professional badminton players. Front Psychol 2016; 7: 1-9.

[33] Zakas A. Bilateral isokinetic peak torque of quadriceps and hamstring muscles in professional soccer players with dominance on one or both two sides. J Sports Med 
481

482

483

484

485
Phys Fitness 2006; 46(1): 28.

[34] Zhang Z. Biomechanical analysis and model development applied to table tennis forehand strokes. Doctoral dissertation, Loughborough University, UK, 2017.

[35] Zhao Q, Lu Y, Jaquess KJ, et al. Utilization of cues in action anticipation in table tennis players. J Sport Sci 2018; 36(23): 2699-2705. 